\title{
Computational Cognitive Linguistics
}

\author{
Jerome A. Feldman \\ ICSI \& UC Berkeley \\ 1947 Center Street \\ Berkeley, CA, 94704 \\ jfeldman@icsi.berkeley.edu
}

\begin{abstract}
1 The talk will describe an ongoing project (modestly named the Neural Theory of Language) that is attempting to model language behavior in a way that is both neurally plausible and computationally practical. The cornerstone of the effort is a formalism called Embodied Construction Grammar (ECG). I will describe the formalism, a robust semantic parser based on it, and a variety of applications of moderate scale. These include a system for understanding the (probabilistic and metaphorical) implications of news stories, and the first cognitively plausible model of how children learn grammar.
\end{abstract}

\section{Introduction}

Computational Linguistics is sometimes narrowly identified with statistical corpus studies, but is much broader. The learning and use of language are inherently information processing tasks and any systematic description of them must be computational. If we want to understand how human brains acquire and exploit language, we are driven to computational theories and models that link neural structure to linguistic behavior. Cognitive Linguistics provides the basic mechanisms for explaining human language processing, but has traditionally been informal and non-computational. Recent work suggests that a formally grounded computational cognitve linguistics can be quite productive.

\section{The Neural Theory of Language}

For some sixteen years, an interdisciplinary group at ICSI and UC Berkeley has been building a theory of language that respects all experimental findings and is consistent with biological and computational constraints. The intellectual base for the project is a synthesis of cognitive linguistics and structured connectionist modelling. This involves work from neurobiology through politics and philosophy (Lakoff 1999). An accessible overview of the goals and methodology of the project can be found in (Regier 1996) and further information is available at:

\section{WWW.icsi.berkeley.edu/NTL}

The focus of this talk is computational; how can an embodied theory of language support scalable systems for language learning and use. The key to scalability in any paradigm is compositionality; our goal in modeling language understanding is to systematically combine the heterogeneous structures posited in cognitive linguistics to yield overall interpretations. We have identified four conceptual primitives that appear to capture the suffice for building scalable language understanding systems: SCHEMA, MAP, (MENTAL) SPACE, and CONSTRUCTION. Schemas are language-independent representation of meanings and constructions are form-meaning pairs. Constructions are form-meaning mappings as depicted in Figure 1. Maps and mental spaces are discussed in (Mok 2004). The ECG formalism has, in addition to the usual inheritance hierarchy, an EVOKES relation that makes an outside structure accessible to a schema through a local name.

As shown in Figure 1, langauge understanding is implemented as having distinct analysis and simulation phases. During analysis, a Semantic Specification (SemSpec) is created from the meaning poles of the constructions, and is essentially a network of schemas with the appropriate roles filled in.. Unification of constructions requires compatability of their embodied semantic scehemas as well as form matching. Crucially, within this network of schemas are executing schemas (or $\mathbf{x}$-schemas Narayanan 1999), which are models of events. They are active structures for event-based asynchronous control that can capture both sequential flow and concurrency.

Simulation is a dynamic process which includes executing the $\mathrm{x}$-schemas specified in the SemSpec and propagating belief updates in a belief network 
(Jensen 1996, Narayanan 1999). This will be discussed further in Section 5.

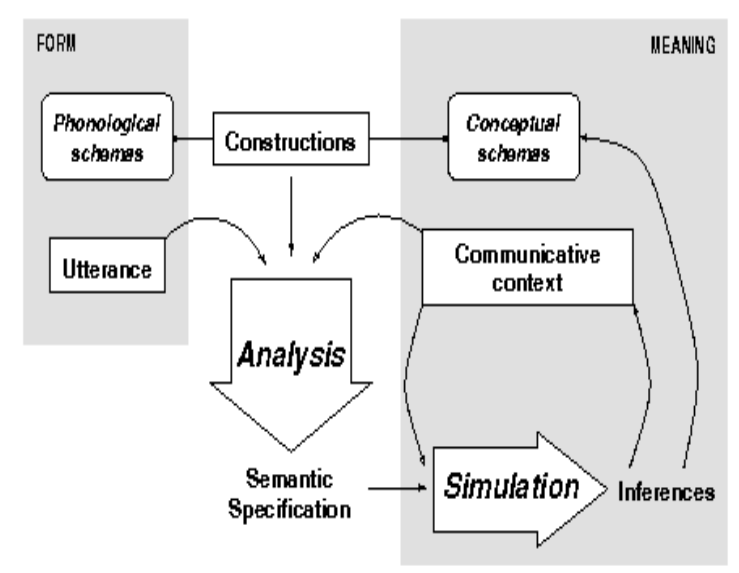

Figure1. Overview of the Comprehension Model

\section{Embodied Construction Grammar}

The cornerstone of the effort is the formalism called Embodied Construction Grammar (ECG). In traditional terms, ECG resembles a unification grammar like HPSG or LFG and many of the computational insights carry over. But the central task of grammar is taken to be accounting for the full range of language learning and use rather than the specification of acceptable forms.

Grammars in ECG are deeply cognitive, with meaning being expressed in terms of cognitive primitives such as image schemas, force dynamics, etc. The hypothesis is that a modest number of universal primitives will suffice to provide the core meaning component for the grammar. Specific knowledge about specialized items, categories and relations will be captured in the external ontology.

As a linguistic formalism, ECG combines the idea of Construction Grammar as form-meaning pairings (Croft 2001, Fillmore \& Kay 1999, Goldberg 1995, etc.) with the embodied semantics of the Cognitive Linguistics tradition (Fauconnier 1997, Lakoff 1999, Langacker 1991, etc.). Computationally, the central ideas involve probabilistic relational models (Pfeffer and Koller 2000, etc.) and active knowledge (Bailey 1998, Narayanan 1999, etc.) along with their reduction to structured connectionist form and thus to neural models (Shastri 2002).

A grammar specification in ECG should simultaneously fill three distinct functions: capturing linguistic insights, specifying the analysis phase of a computational system, and serving as the high level description of the neural embodiment of the grammar. This has been achieved in some simple cases and serves as a major constraint in all ECG efforts.

The deep semantic construction grammar of ECG also supports a novel style of general robust parsing. The first phase of analysis is an modified chunk parser (Abney 1996).

The chunker generates a set of semantic chunks stored in a chart. The second phase of analysis extracts the smallest number of chunks that span the utterance from the chart, and performs semantic integration. Their common semantic structures are merged, and the resulting analyses are ranked according to the semantic density metric

Without a complete analysis of an utterance, the system must infer exactly how a set of local, partial semantic structures fit together into a

coherent, global analysis of the utterance. The approach taken is an abductive one in that it assumes compatible structures are the same and merges them. This has been shown to work quite well in a system for modeling the learning of new constructions (Bryant 2004, Chang 2004).

\section{Applications}

Narayanan (Narayan 1999) has built a biologically plausible model of how such metaphorical uses can be understood by mapping to their underlying embodied meaning. We assume that people can execute $\mathrm{x}$-schemas with respect to structures that are not linked to the body, the here and the now. In this case, $\mathrm{x}$-schema actions are not carried out directly, but instead trigger simulations of what they would do in the imagined situation. This ability to simulate or imagine situations is a core component of human intelligence and is central to our model of language. The system models the physical world as other $\mathrm{x}$-schemas that have input/output links to the $\mathrm{x}$-schema representing the planned action.

In the computational implementation, the spatial motion domain (source domain) is encoded as connected $x$-schemas. Our model of the source domain is a dynamic system based on inter- $x$ schema activation, inhibition and interruption. In the simulation framework, whenever an executing $\mathrm{x}$-schema makes a control transition, it potentially modifies state, leading to asynchronous and parallel triggering or inhibition of other x-schemas. The notion of system state as a graph marking is inherently distributed over the network, so the 
working memory of an x-schema-based inference system is distributed over the entire set of $x-$ schemas and source domain feature structures. The KARMA system has been tested on narratives from the abstract domain of international economics. The implemented model has about 100 linked $\mathrm{x}$-schemas, and about 50 metaphor maps from the domains of health and spatial motion. These were developed using a database of 30 2-3 phrase fragments from newspaper stories all of which have been successfully interpreted by the program. Results of testing the system have shown that a surprising variety of fairly subtle and informative inferences arise from the interaction of the metaphoric projection of embodied terms with the topic specific target domain structure (Narayanan, 1999). Among the inferences made were those related to goals (their accomplishment, modification, subsumption, concordance, or thwarting), resources (consumption, production, depletion, level), aspect (temporal structure of events) frame-based inferences, perspectival inferences, and inferences about communicative intent.

The ECG formalisms as well as the analyzer described above play a crucial role in a computational model of how language comprehension may drive the acquisition of early phrasal and clausal constructions (Chang, 2004). This model takes ECG as the target representation to be learned from a sequence of utterances in context. Learning is usage-based in that utterances are first analyzed using the existing set of constructions, typically resulting in only a partial analysis that neither provides complete coverage of the richer background context nor exploits all the potential input forms and relations in the utterance. This incomplete analysis prompts the formation of new constructions that take up the slack. Constructions can also be formed on the basis of similarity (two constructions can merge into a more general construction) and co-occurrence (two constructions can compose into a larger construction).

Besides specifying the means for forming new ECG constructions, the acquisition model provides an overarching computational framework for converging on an optimal set of constructions, based on a minimum description length principle ( Rissanen 1978) that favors compactness in describing both the grammar and the statistical properties of the data. This framework extends previous work in Bayesian model merging for lexical acquisition (Bailey, 1997) and the induction of context-free grammars (Stolcke 1994) to handle the relational structures and usage-based considerations madepossible with ECG. Specifically, the criteria employed favor constructions that have simple descriptions (relative to the available meaning representations and current set of constructions) and are frequently employed.

The model has been applied to learn simple English motion constructions from a corpus of child-directed utterances, paired with situation representations. The resulting learning trends reflect cross-linguistic acquisition patterns, including the incremental growth of the constructional inventory based on experience, the prevalence of early grammatical markers for

conceptually basic scenes (Slobin, 1985) and the learning of lexically specific verb island constructions before more abstract grammatical patterns (Tomasello, 1992). For current purposes, the systems described demonstrate the utility of the ECG formalism for supporting computational modeling and offers a precisely specified instantiation of the simulation-based approach to language.

\section{Conclusion}

For current purposes, the systems described above demonstrate the utility of the ECG formalism for supporting computational modeling and offer a precisely specified instantiation of the simulation-based approach to language.

Cognitive Linguistics has developed many profound insights, but these had not been formalized and made computationally tractable. Recent results like these suggest that a Computational Cognitive Linguistics is both scientifically productive and a semantic basis for a wide range of natural language understanding applications.

\section{Acknowledgements}

The NTL project has always been a team effort. Special thanks for this presentation go to John Bryant, Nancy Chang, Eva Mok, and Srini Narayanan.

\section{References}

S. Abney. 1996. Partial parsing via finite-state cascades. In Workshop on Robust Parsing, $8^{\text {th }}$ European Summer School in Logic, Language and Information, pages 8-15, Prague, Czech Republic. 
D. Bailey, N. Chang, J. Feldman and S. Naryanan. 1998. Extending embodied lexical development. In Proceedings of the twentieth annual meeting of the Cognitive Science Society COGSCI-98, Madison.

B. Bergen and N. Chang. 2002. Embodied construction grammar in simulation-based language understanding. Technical Report TR-02004, ICSI, 2002. To appear in Oestman and Reid, eds., Construction Grammar(s): Cognitive and Cross-Linguistic Dimensions. John Benjamins.

B. Bergen, N. Chang and S. Narayan. 2004. Simulated Action in an Embodied Construction Grammar. Proc. 26th Cognitive Science Society Conference.

J. Bryant. 2003. Constructional analysis. Master's thesis, UC Berkeley.

J. Bryant. 2004. Towards Cognitive, Compositional Construction Grammar. ROMAND 2004: RObust Methods in Analysis of Natural language Data, Geneva.

G. Buccino, F. Binkofski, G. R. Fink, L. Fadiga, L. Fogassi, V. Gallese, R. J. Seitz, K Zilles, G. Rizzolatti and H.-J. Freund. 2001. Action observation activates premotor and paretal areas in a somatotopic manner: An fMRI study. European Journal of Neuroscience, 13:400-404.

N. Chang. 2004a. Constructing Grammar: $A$ computational model of the emergence of early constructions. $\mathrm{PhD}$ thesis, University of California at Berkeley.

N. Chang. 2004b. Putting Meaning into Grammar Learning. In Proc. Psychocomputational Models of Human Language Acquisition. Geneva.

N. Chang, J. Feldman, R. Porzel and K. Sanders. 2002. Scaling cognitive linguistics: Formalisms for language understanding. In Proceedings of the $1^{\text {st }}$ International Workshop on Scalable Natural Language Understanding, Heidelberg, Germany.

N. Chang and O. Gurevich. Context-driven construction learning. Proc. 26th Cognitive Science Society Conference.

N. Chang, S. Narayanan and M. R. L. Petruck. 2002. Putting frames in perspective. In Proceedings of the nineteenth international conference on Computational Linguistics (COLING 2002), Taipei.

W. Croft. 2001. Radical Construction Grammar. Oxford University Press.

G. Fauconnier. 1997. Mappings in Thought and Language. New York: Cambridge University Press.

J. Feldman. 2002. The meaning of reference in embodied construction grammar. Technical Report, International Computer Science Institute, ICSI TR-02-11, Berkeley, CA.

J. Feldman. 2004. From Molecule to Metaphor: A Neural Theory of Language. Forthcoming

J. Feldman and S. Narayanan. 2003. Embodied meaning in a neural theory of language. Brain and Language, 89:385-392.

C. Fillmore and P. Kay. 1999. Construction Grammar. CSLI, Stanford, CA to appear.

A. E. Goldberg. 1995. Constructions: A Construction Grammar Approach to Argument Structure. University of Chicago Press.

F. Jensen. 1996. An Introduction to Bayesian Networks. Berlin: Springer Verlag.

G. Lakoff and M. Johnson. 1999. Philosophy in the flesh: The embodied mind and its challenge to western thought, $2^{\text {nd }}$ ed. 202-251. Cambridge: Cambridge University Press.

R. Langacker. 1991. Concept, Image, Symbol: The Cognitive Basics of Grammar. Mouton de Gruyter.

B. MacWhinney. 1991. The CHILDES project: Tools for analyzing talk. Erlbaum Associates, Mahwah, New Jersey.

E. Mok, J. Bryant, J. Feldman. 2004. Scaling up to Mental Spaces. In Proceedings of the Second International Workshop on Scalable Natural Language Understanding, Boston.

S. Narayanan. 1999. Moving right along: A computational model of metaphoric reasoning about events. In Proceedings of the national Conference on Artificial Intelligence AAAI-99. Orlando, FL.

S. Narayanan and S. Harabagiu (2004). Question Answering Based on Semantic Structures, $21^{\text {st }}$ 
International Conference on Computational Linguistics (COLING 2004), Geneva, Switzerland (to appear).

F. Pulvermueller. 2001. Brain reflections of words and their meaning. Trends in Cognitive Science, 5(12).

T. Regier. 1996. The Human Semantic Potential. MIT Press, Cambridge, MA.

A. Pfeffer and D. Koller. Semantics and Inference for Rewcursive Probability Models. National Conference on Artificial Intelligence (AAAI), 2000.

J. Rissanen. 1978. Modeling by shortest data description. Automatica, 14:465-471.

L. Shastri. 2002. episodic memory and corticohippocampal interactions. Trends in Cognitive Science, 6:162-168.

D.I. Slobin. 1985. Crosslinguistic evidence for the language-making capacity. In Slobin, D.I., editor, Theoretical Issues, volume 2 of The Crosslinguistic Study of Language Acquisition, chapter 15. Lawrence Erlbaum Associates, Mahwah, New Jersey.

A. Stolcke 1994 Bayesian Learning of Probabilistic Language Models. Doctoral thesis, UC Berkeley.

M. Tomasello. 1992. First verbs: A case study of early grammatical development. Cambridge University Press, Cambridge, UK.

M. Tomasello. 2003. Constructing a Language: A usage-Based Theory of Language Acquisition. Harvard University Press, Cambridge, MA. 